\title{
Indirubin-3'-oxime impairs mitochondrial oxidative phosphorylation and prevents mitochondrial permeability transition induction
}

\author{
Ana T. Varela ${ }^{1}$, Ana P. Gomes ${ }^{1}$, Anabela M. Simões, João S. Teodoro, Filipe V. Duarte, \\ Anabela P. Rolo, Carlos M. Palmeira* \\ Center for Neurosciences and Cell Biology, Department of Zoology, University of Coimbra, 3004-517 Coimbra, Portugal
}

\section{A R T I C L E I N F O}

\section{Article history:}

Received 5 May 2008

Revised 24 July 2008

Accepted 5 August 2008

Available online 14 August 2008

\section{Keywords:}

Indirubin-3'-oxime

Mitochondria

Oxidative phosphorylation

Mitochondrial permeability transition

\begin{abstract}
A B S T R A C T
Indirubin, a red colored 3,2'-bisindole isomer, is a component of Indigo naturalis and is an active ingredient used in traditional Chinese medicine for the treatment of chronic diseases. The family of indirubin derivatives, such as indirubin-3'-oxime, has been suggested for various therapeutic indications. However, potential toxic interactions such as indirubin effects on mitochondrial bioenergetics are still unknown. This study evaluated the action of indirubin-3'-oxime on the function of isolated rat liver mitochondria contributing to a better understanding of the biochemical mechanisms underlying the multiple effects of indirubin. Indirubin-3'-oxime incubated with isolated rat liver mitochondria, at concentrations above $10 \mu \mathrm{M}$, significantly depresses the phosphorylation efficiency of mitochondria as inferred from the decrease in the respiratory control and $\mathrm{ADP} / \mathrm{O}$ ratios, the perturbations in mitochondrial membrane potential and in the phosphorylative cycle induced by ADP. Furthermore, indirubin-3'-oxime at up to $25 \mu \mathrm{M}$ stimulates the rate of state 4 respiration and inhibits state 3 respiration. The increased lag phase of repolarization was associated with a direct inhibition of the mitochondrial ATPase. Indirubin-3'-oxime significantly inhibited the activity of complex II and IV thus explaining the decreased FCCP-stimulated mitochondrial respiration. Mitochondria pre-incubated with indirubin-3'-oxime exhibits decreased susceptibility to calcium-induced mitochondrial permeability transition. This work shows for the first time multiple effects of indirubin-3'-oxime on mitochondrial bioenergetics thus indicating a potential mechanism for indirubin-3'-oxime effects on cell function.
\end{abstract}

(c) 2008 Elsevier Inc. All rights reserved.

\section{Introduction}

Indirubin, a red colored 3,2'-bisindole isomer, is a component of Indigo naturalis and is an active ingredient used in traditional Chinese medicine for the treatment of chronic diseases such as leukemias (Eisenbrand et al., 2004). Several papers describing the properties of the indirubin family as cyclin-dependent kinases (CDKs) (Hoessel et al.,

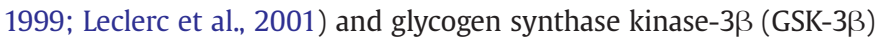
(Leclerc et al., 2001; Meijer et al., 2003) inhibitors unravelled the potential therapeutic indication of these compounds. Indirubin and derivatives may have important implications for the development of therapies for many diseases such as ischemia-reperfusion, Alzheimer's disease, cancer and type 2 diabetes (Barillas et al., 2007; Meijer et al., 2003; Jope et al., 2007), as well as in stem cell therapy (Sato et al., 2004).

Numerous indirubin analogs have been synthesized to optimize this promising drug scaffold. Indirubin-3'-oxime is an analogue of indirubin commercially available. Addition of a 3-oxime substitution

\footnotetext{
* Corresponding author. Fax: +351 239855789

E-mail address: palmeira@ci.uc.pt (C.M. Palmeira)

${ }^{1}$ Both authors contributed equally to the work.
}

led to an overall increase in kinase inhibitory effects (Zhang et al., 2006) and increased solubility (Meijer et al., 2003). However, drugs for various therapeutic indications frequently have unexpected effects as a result from unknown interactions between the intended drug and biochemical pathways. Such unexpected activities may lead to adverse effects and toxicity, thus disabling the potential therapeutic action. In the last years, several mitochondrial off targets of drug action have been shown as responsible for adverse effects. Such mitochondrial toxicity leads to metabolic failure since mitochondria constitute the principal energy-producing organelles of the cell through oxidative phosphorylation. Therefore, alterations of mitochondrial bioenergetic features by mitochondrial toxicants perturb energetic charge and balance of cell and may cause drastic consequences on cellular function.

Previous studies suggest an activity of indirubin and its derivates on the mitochondria (Lee et al., 2005 and MacDonald et al., 2006), however the effect of indirubin in mitochondrial bioenergetics remains unknown. So, this study examines the effects of indirubin-3'-oxime in bioenergetic functions of isolated rat liver mitochondria contributing to a better comprehension of biochemical mechanisms underlying the effects of indirubin. By using a cell-free model such as isolated mitochondria as the experimental model it is possibly to clearly identify if indirubin-3'- 
A
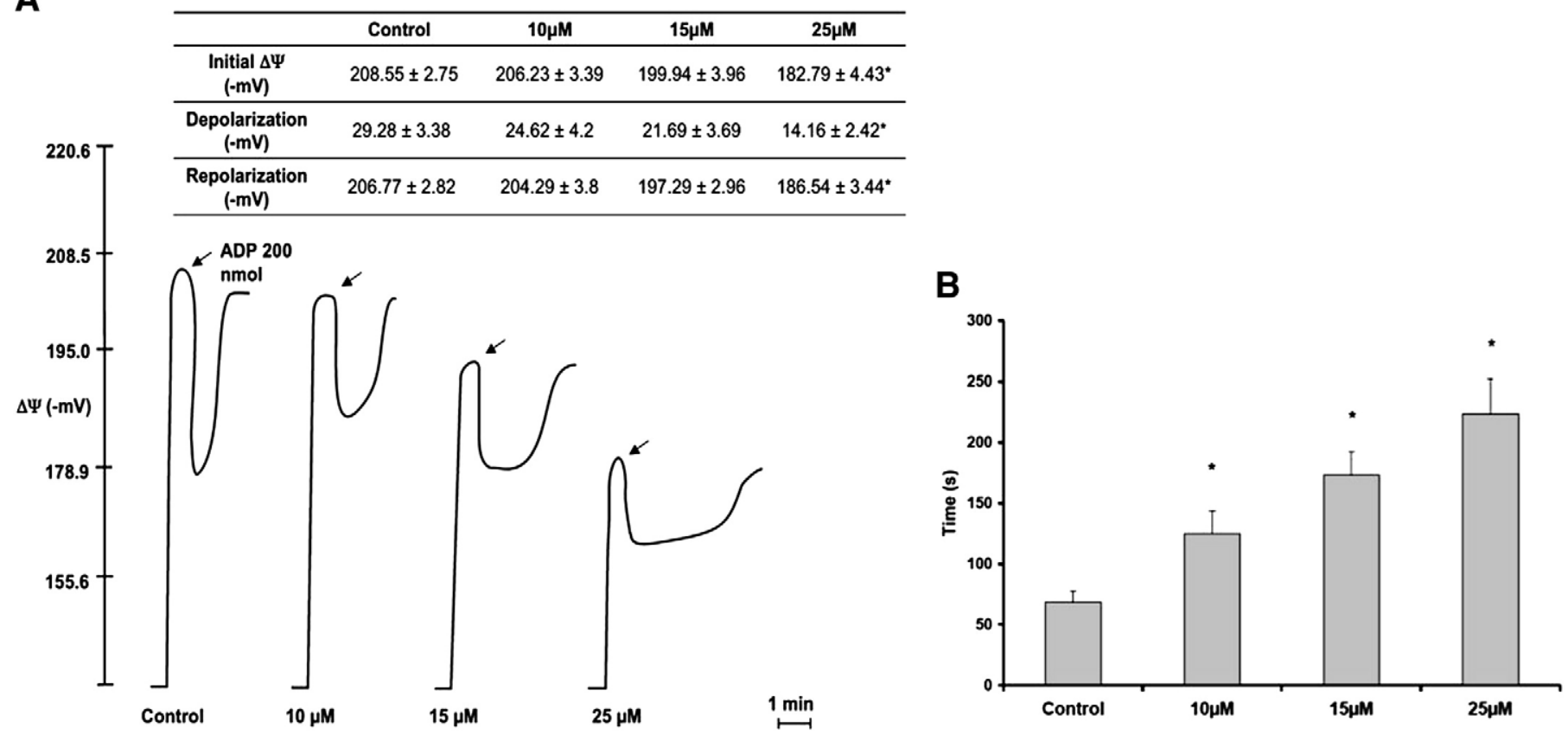

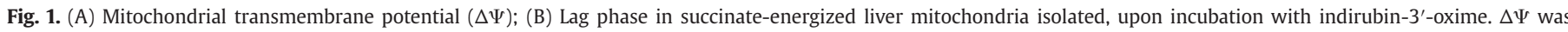

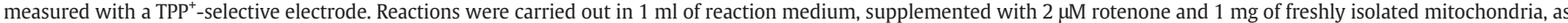

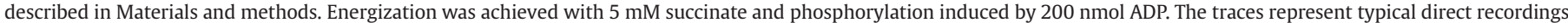
and data are means \pm S.E.M of experiments performed with four different mitochondrial preparations. $*$ indicates statistically significant difference versus control $(P<0.05)$.
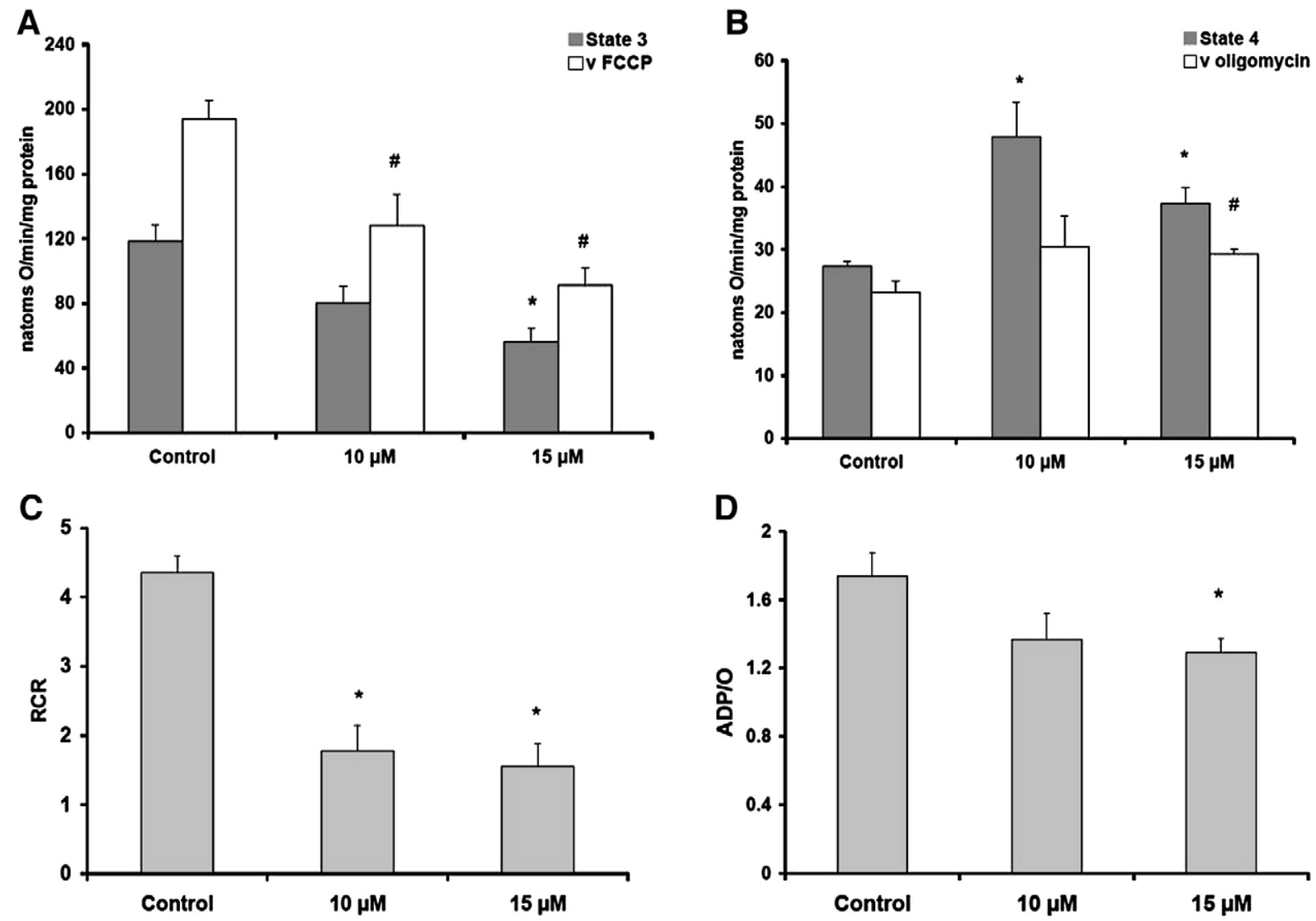

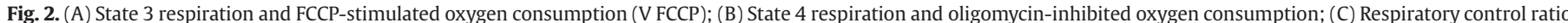

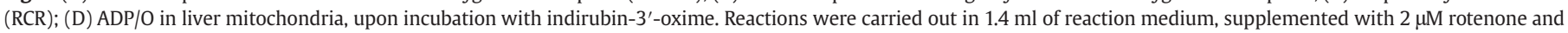

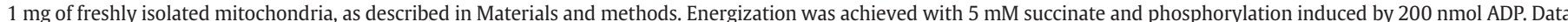

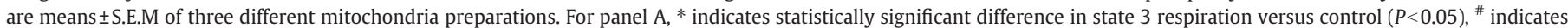

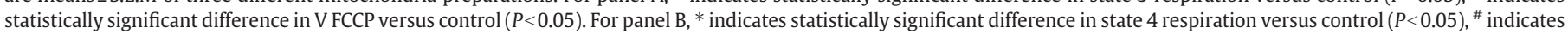
statistically significant difference in $\mathrm{V}$ Oligomycin versus control $(P<0.05)$. For panels $C$ and $\mathrm{D}$, * indicates statistically significant difference versus control $(P<0.05)$. 
oxime is a mitochondrial toxicant by direct disruption of oxidative phosphorylation or interference with the oxidation of reducing substrates that deliver electrons to the respiratory chain.

\section{Materials and methods}

Chemicals. All compounds were purchased from Sigma Chemical Co. (St. Louis, MO). All other reagents and chemicals used were the highest grade of purity commercially available.

Incubations with indirubin-3'-oxime. Stock solutions of indirubin-3'oxime were prepared in DMSO. Mitochondria were incubated with several concentrations of indirubin- $3^{\prime}$-oxime at $25^{\circ} \mathrm{C}$ for $3 \mathrm{~min}$. Similar concentrations of DMSO were added to control preparations in order to exclude solvent effect. The final concentration of DMSO was less than $0.01 \%$.

Preparation of liver mitochondria. Mitochondria were isolated from the liver of male Wistar rats by conventional methods (Gazotti et al., 1979) with slight modifications (Rolo et al., 2000). Protein content was determined by the biuret method (Gornall et al., 1949) calibrated with bovine serum albumin.

Mitochondrial respiration. Oxygen consumption of isolated mitochondria was polarographically determined with a Clark oxygen electrode (Estabrook, 1967), as previously described (Rolo et al., 2000). Mitochondria (1 mg) were suspended under constant stirring, at $25^{\circ} \mathrm{C}$, in $1.4 \mathrm{ml}$ of standard respiratory medium ( $130 \mathrm{mM}$ sucrose, 50 $\mathrm{mM} \mathrm{KCl}, 5 \mathrm{mM} \mathrm{MgCl}_{2}, 5 \mathrm{mM} \mathrm{KH}_{2} \mathrm{PO}_{4}, 50 \mu \mathrm{M}$ EDTA, and 5 mM HEPES ( $\mathrm{pH}$ 7.4) and $2 \mu \mathrm{M}$ rotenone). Indirubin-3'-oxime was added to the respiratory medium with mitochondria and allowed to incubate for 3 min before the addition of succinate $(5 \mathrm{mM})$. State 3 respiration was induced by adding $200 \mathrm{nmol}$ ADP. The oxygen consumption was also measured in the presence of oligomycin $(0.5 \mu \mathrm{g} / \mathrm{mg}$ protein $)$ and $1 \mu \mathrm{M}$ carbonylcyanide-p-trifluoromethoxyphenylhydrazon (FCCP).

Membrane potential $(\Delta \Psi)$ measurements. $\quad \Delta \Psi$ was estimated using an ion-selective electrode to measure the distribution of tetraphenylphosphorium $\left(\mathrm{TPP}^{+}\right)$according to previously established methods (Kamo et al., 1979 and Palmeira et al., 1994). Mitochondria ( $1 \mathrm{mg}$ ) were suspended in $1 \mathrm{ml}$ of standard respiratory medium (as in mitochondrial respiration) supplemented with $3 \mu \mathrm{M} \mathrm{TPP}$. Indirubin-3'-oxime was added to the respiratory medium with mitochondria and $\mathrm{TPP}^{+}$and allowed to incubate for 3 min before the

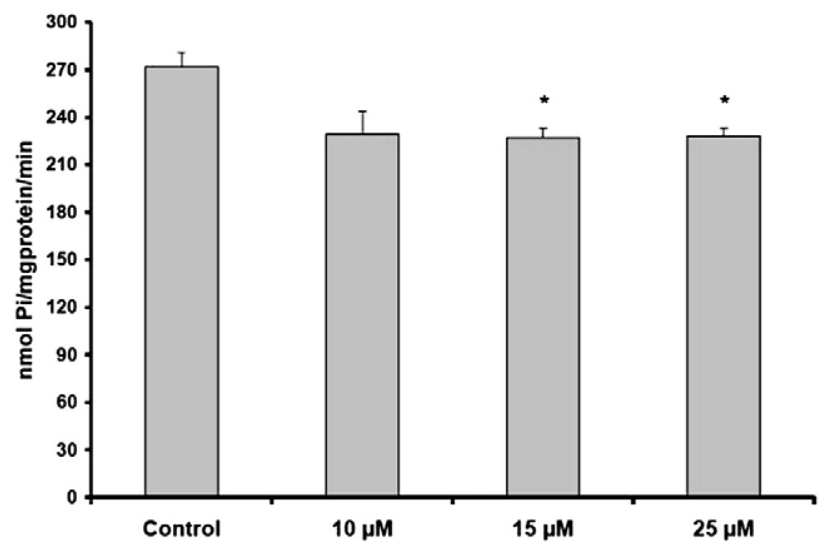

Fig. 3. ATPase activity in liver mitochondria incubated with indirubin-3'-oxime. ATPase activity was evaluated spectrophotometrically at $660 \mathrm{~nm}$, in association with ATP hydrolysis. Reactions were carried out in $2 \mathrm{ml}$ of reaction medium, supplemented with $0.25 \mathrm{mg}$ of freeze-thawed mitochondria, as described in Materials and methods. Data are means \pm S.E.M of three different mitochondrial preparations. * indicates statistically significant difference versus control $(P<0.05)$ addition of succinate $(5 \mathrm{mM})$. A matrix volume of $1.1 \mu \mathrm{l} / \mathrm{mg}$ protein was assumed.

Measurement of the mitochondrial permeability transition. Mitochondrial swelling was estimated by changes in light scattering, as monitored spectrophotometrically at $540 \mathrm{~nm}$ (Palmeira and Wallace, 1997). The incubation medium contained $200 \mathrm{mM}$ sucrose, $10 \mathrm{mM}$ TrisMops ( $\mathrm{pH} 7.4$ ), $1 \mathrm{mM} \mathrm{KH}_{2} \mathrm{PO}_{4}$, and $10 \mu \mathrm{M}$ EGTA supplemented with $2 \mu \mathrm{M}$ rotenone and $5 \mathrm{mM}$ succinate. The reaction was stirred continuously and the temperature maintained at $25{ }^{\circ} \mathrm{C}$. The experiments were started by the addition of $1 \mathrm{mg}$ of mitochondria (final volume $2 \mathrm{ml}$ ). Calcium was added after incubation for $3 \mathrm{~min}$ of indirubin-3'-oxime with mitochondria.

ATPase activity. ATPase activity was determined spectrophotometrically at $660 \mathrm{~nm}$, in association with ATP hydrolysis. The reaction was carried out at $37^{\circ} \mathrm{C}$, in $2 \mathrm{ml}$ reaction medium (125 mM sucrose, $65 \mathrm{mM} \mathrm{KCl}, 2.5 \mathrm{mM} \mathrm{MgCl}_{2}$ and $0.5 \mathrm{mM}$ HEPES, pH 7.4). After the addition of freeze-thawed mitochondria $(0.25 \mathrm{mg})$, indirubin-3'-oxime was added and allowed to incubate for $3 \mathrm{~min}$ before the initiation of reaction with the addition of $2 \mathrm{mM} \mathrm{Mg}^{2+}$-ATP, in the presence or absence of oligomycin ( $1 \mu \mathrm{g} / \mathrm{mg}$ protein). After $10 \mathrm{~min}$, the reaction was stopped by adding $1 \mathrm{ml}$ of $40 \%$ trichloroacetic acid and the samples centrifuged for $5 \mathrm{~min}$ at $3000 \mathrm{rpm} .2 \mathrm{ml}$ of ammonium molybdate plus $2 \mathrm{ml} \mathrm{dH}_{2} \mathrm{O}$ were then added to $1 \mathrm{ml}$ of supernatant. ATPase activity was calculated as the difference in total absorbance and absorbance in the presence of oligomycin.

Cytochrome c oxidase activity. Cytochrome $c$ oxidase activity was polarographically determined, as previously described (Brautigan et al., 1978). The reaction was carried out at $25{ }^{\circ} \mathrm{C}$ in $1.4 \mathrm{ml}$ of standard respiratory medium (as in mitochondrial respiration) supplemented with $2 \mu \mathrm{M}$ rotenone, $10 \mu \mathrm{M}$ oxidized cytochrome $c$ and $0.3 \mathrm{mg}$ Triton $\mathrm{X}-100$. After the addition of freeze-thawed mitochondria $(0.5 \mathrm{mg})$, indirubin-3'-oxime was added and allowed to incubate for $3 \mathrm{~min}$ before the initiation of the reaction by adding $5 \mathrm{mM}$ ascorbate plus $0.25 \mathrm{mM}$ tetramethylphenylene-diamine (TMPD).

Succinate dehydrogenase activity. Succinate dehydrogenase activity was polarographically determined as previously described (Singer, 1994). The reaction was carried out at $25{ }^{\circ} \mathrm{C}$ in $1.4 \mathrm{ml}$ of standard respiratory medium (as in mitochondrial respiration) supplemented with $5 \mathrm{mM}$ succinate, $2 \mu \mathrm{M}$ rotenone, $0.1 \mu \mathrm{g}$ antimycin $\mathrm{A}, 1 \mathrm{mM} \mathrm{KCN}$ and $0.3 \mathrm{mg}$ Triton X-100. After the addition of freeze-thawed mitochondria $(0.5 \mathrm{mg})$, indirubin-3'-oxime was added and allowed to incubate for $3 \mathrm{~min}$ before the initiation of reaction with $1 \mathrm{mM}$ phenazinemetasulphate (PMS).

Measurement of mitochondrial calcium fluxes. The accumulation and release of calcium by isolated rat liver mitochondria were determinate using a calcium-sensitive fluorescent dye, Calcium Green-5 N (Rajdev and Reynolds, 1993). The reactions were carried out at $25^{\circ} \mathrm{C}$, in $2 \mathrm{ml}$ of standard incubation medium (200 mM sucrose, $10 \mathrm{mM}$ Tris-MOPS ( $\mathrm{pH}$ 7.4), $1 \mathrm{mM} \mathrm{KH}_{2} \mathrm{PO}_{4}$ and $10 \mu \mathrm{M} \mathrm{EGTA}$ ), supplemented with $2 \mu \mathrm{M}$ rotenone and $0.5 \mu \mathrm{g} / \mathrm{mg}$ protein of oligomycin. Free calcium was monitored with $100 \mathrm{nM}$ calcium Green 5-N. Mitochondria (1 mg) were suspended in twelve well plates. Energization was obtained with succinate $(5 \mathrm{mM})$. Fluorescence was recorded continuously using a Perkin-Elmer VICTOR 3 , with excitation and emission wavelengths of 485 and 535, respectively. Calcium fluxes are expressed as relative fluorescence units (RFU). At the concentrations used, indirubin-3'-oxime did not interfere with the experimental assay.

Statistical analysis. Results are presented as mean \pm SEM. Statistical evaluation was performed using two-tails Student's $t$ test. A $P$ value $<0.05$ was considered statistically significant. 


\section{Results}

Effects of indirubin-3'-oxime in mitochondrial transmembrane potential

Taking into account the fundamental role of mitochondrial transmembrane potential for the phenomenon of oxidative phosphorylation, $\Delta \Psi$ was evaluated in succinate-energized mitochondria (Fig. 1A). $\Delta \Psi$ was decreased in mitochondria pre-incubated with indirubin-3'-oxime $10 \mu \mathrm{M}, 15 \mu \mathrm{M}$ and $25 \mu \mathrm{M}$, being statistically significant for the concentration of $25 \mu \mathrm{M}$.

ADP-induced depolarization and $\Delta \Psi$ after repolarization (mitochondrial capacity to establish $\Delta \Psi$ after ADP phosphorylation) was also decreased in mitochondria pre-incubated with indirubin-3'oxime, but again it was only statistically significant for the concentration of $25 \mu \mathrm{M}$. No differences were observed in mitochondrial transmembrane potential in mitochondria pre-incubated with indirubin-3'-oxime $5 \mu \mathrm{M}$ (data not shown).

The lag phase (time necessary for ADP phosphorylation) was significantly enlarged when mitochondria was incubated $10 \mu \mathrm{M}, 15 \mu \mathrm{M}$ and $25 \mu \mathrm{M}$ with indirubin-3'-oxime (Fig. 1B).

\section{Effects of indirubin-3'-oxime in mitochondrial respiration}

Oxidative phosphorylation capacity was investigated by following oxygen consumption upon succinate oxidation. Mitochondrial state 3 respiration (ADP-induced oxygen consumption) was significant decreased in mitochondria pre-incubated with indirubin-3'-oxime 15 $\mu \mathrm{M}$. Similarly, oxygen consumption stimulated by FCCP, a well known respiratory chain uncoupler, was significant decreased in mitochondria pre-incubated with indirubin-3'-oxime $10 \mu \mathrm{M}$ and $15 \mu \mathrm{M}$ (Fig. 2A).

The consumption of oxygen after ADP phosphorylation (state 4 respiration) was increased in mitochondria pre-incubated with indirubin-3'-oxime $10 \mu \mathrm{M}$ and $15 \mu \mathrm{M}$ when compared with control (Fig. 2B). Mitochondrial respiration in the presence of oligomycin, a known inhibitor of the mitochondrial $F_{1} F_{0}$-ATPsynthase, was increased in mitochondria pre-incubated with indirubin-3'-oxime $10 \mu \mathrm{M}$ and $15 \mu \mathrm{M}$, being statistically significant for the concentration of $15 \mu \mathrm{M}$ (Fig. 2B).

The ratio between mitochondrial state 3 and state 4 respiration (RCR), was significantly decreased in mitochondria pre-incubated with indirubin-3'-oxime $10 \mu \mathrm{M}$ and $15 \mu \mathrm{M}$ (Fig. 2C). The ADP/O ratio, an indicator of oxidative phosphorylation efficiency, showed a tendency to decrease when mitochondria was incubated with indirubin-3'-oxime. However, this decrease was statistically significant only for $15 \mu \mathrm{M}$ (Fig. 2D).

\section{Effects of indirubin-3'-oxime in mitochondrial ATPase activity}

The decreased performance of phosphorylation in mitochondria pre-incubated with indirubin-3'-oxime, as reflected by an increased lag phase and an increase in mitochondrial respiration in the presence of oligomycin, suggested alterations in the $\mathrm{F}_{1} \mathrm{~F}_{0}$-ATPsynthase, a key component of the phosphorylative system. ATPase activity was decreased in mitochondria pre-incubated with indirubin-3'-oxime with $10 \mu \mathrm{M}, 15 \mu \mathrm{M}$ and $25 \mu \mathrm{M}$, being this decrease statistically significant for $15 \mu \mathrm{M}$ and $25 \mu \mathrm{M}$ (Fig. 3).

Effects of indirubin-3'-oxime on succinate dehydrogenase and cytochrome c oxidase activities

The decreased rate of oxygen consumption induced by FCCP in mitochondria pre-incubated with indirubin-3'-oxime, suggested alterations in the mitochondrial electron transport chain complexes. In view of this, succinate dehydrogenase and cytochrome $c$ oxidase activities were evaluated. Mitochondria pre-incubated with indirubin3'-oxime $15 \mu \mathrm{M}, 25 \mu \mathrm{M}$ and $50 \mu \mathrm{M}$ exhibited decreased activities in both of these enzymes, while mitochondria pre-incubated with indirubin3'-oxime $5 \mu \mathrm{M}$ and $10 \mu \mathrm{M}$, didn't show significant differences in activities of succinate dehydrogenase and cytochrome $c$ oxidase, comparatively to control (Figs. $4 \mathrm{~A}$ and $\mathrm{B}$ ).

\section{Effects of indirubin-3'-oxime on the induction of the mitochondrial permeability transition}

Since mitochondria possess a finite capacity for accumulating calcium before undergoing the MPT, calcium-induced mitochondrial swelling was evaluated. Mitochondria pre-incubated with indirubin3'-oxime were less susceptible to undergo calcium-dependent mitochondrial swelling, comparatively to control (Fig. 5A). Mitochondria pre-incubated with indirubin-3'-oxime and incubated with $100 \mu \mathrm{M}$ ADP revealed an increased threshold in calcium-induced swelling comparatively to mitochondria incubated only with $100 \mu \mathrm{M}$ ADP (Fig. 5B). Pre-treatment with $1 \mu \mathrm{M}$ CyA completely prevented calciumdependent mitochondrial swelling, indicating that the decreased in absorbance was the result of the calcium-induced MPT.

\section{Effects of indirubin-3'-oxime on mitochondrial calcium flux}

Since mitochondria pre-incubated with indirubin-3'-oxime decreased the susceptibility to calcium-induced swelling, mitochondrial calcium fluxes were evaluated. Calcium uptake by mitochondria was identical in both control and in mitochondria pre-incubated with indirubin-3'-oxime. In control experiment, calcium that was taken by

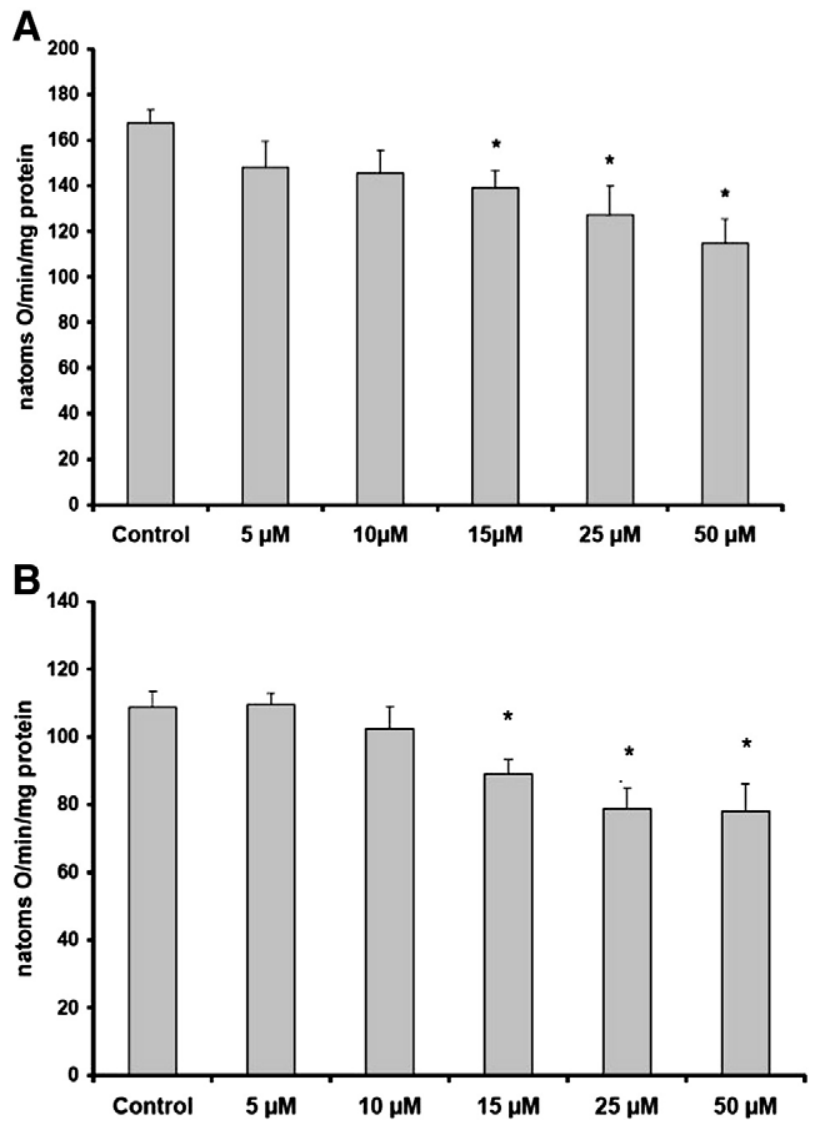

Fig. 4. (A) Cytochrome $c$ oxidase activity; (B) Succinate dehydrogenase activity in liver mitochondria incubated with indirubin-3'-oxime. Enzyme activity was polarographically determined with a Clark oxygen electrode. Mitochondria $(0.5 \mathrm{mg})$ were suspended under constant stirring, at $25^{\circ} \mathrm{C}$, in $1.4 \mathrm{ml}$ of standard respiratory medium. The reaction media was supplemented with $2 \mu \mathrm{M}$ rotenone, $10 \mu \mathrm{M}$ cytochrome $c, 1 \mathrm{mM} \mathrm{KCN}, 0,3 \mathrm{mg}$ Triton X-100 and the reaction initiated by adding $0.25 \mathrm{mM}$ ascorbate/TMPD. Data expressed as means \pm SEM of three different mitochondrial preparations. For each panel, $*$ indicates statistically significant difference versus control $(P<0.05)$. 

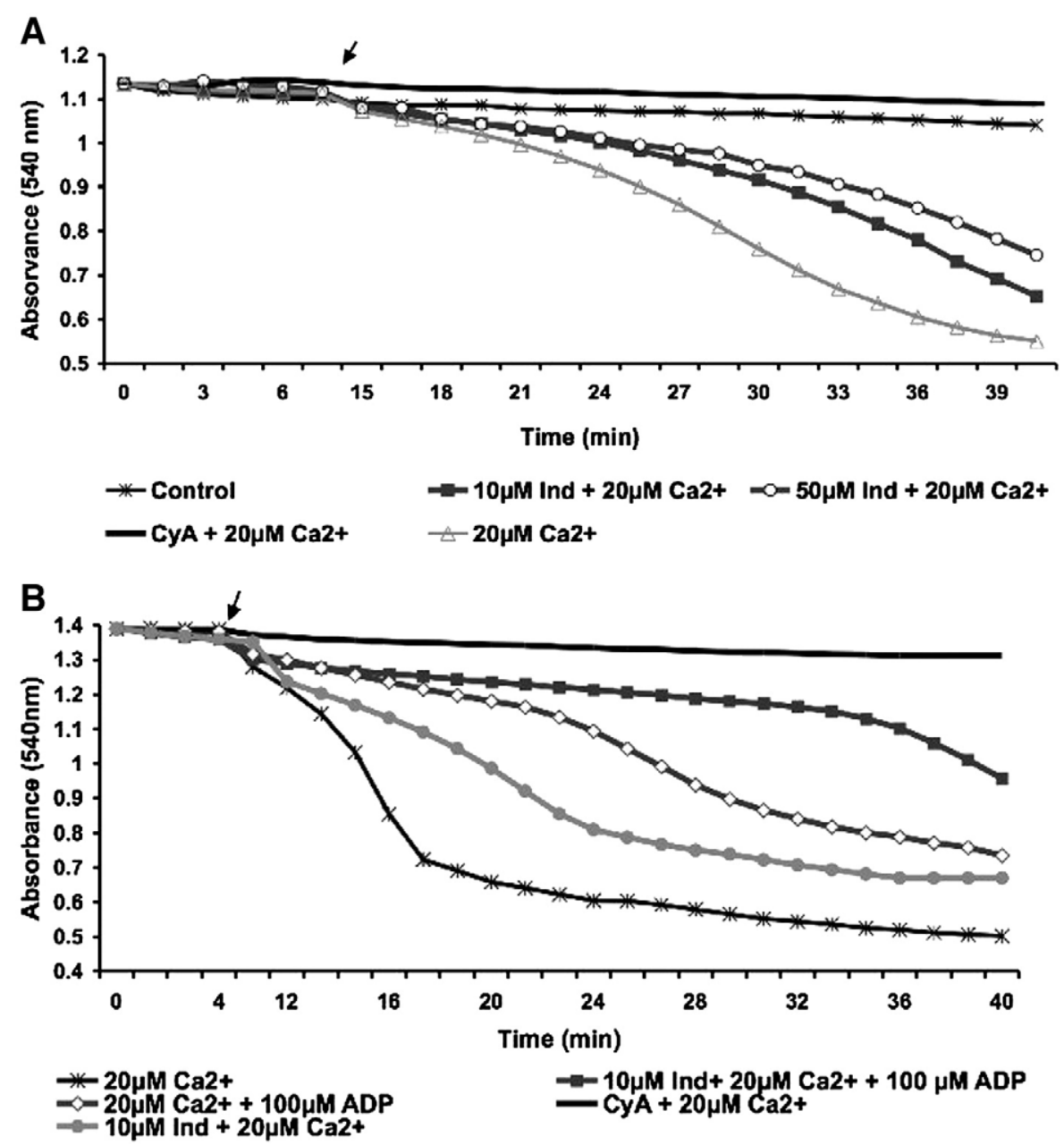

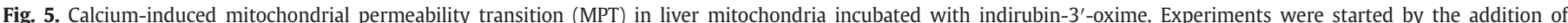

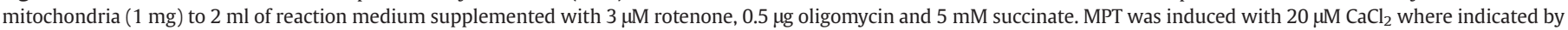

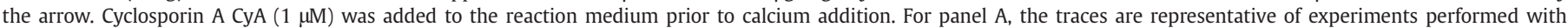

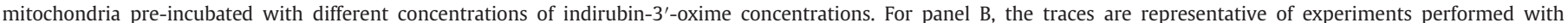
mitochondria pre-incubated with $10 \mu \mathrm{M}$ indirubin-3'-oxime and $100 \mathrm{nmol}$ ADP.

mitochondria after energization with succinate, was retained during approximately $13 \mathrm{~min}$. After this period of time, calcium was released by MPT induction, as demonstrated by pre-treatment with $1 \mu \mathrm{M} \mathrm{CyA}$, which completely prevented calcium release (Fig. 6). Pre-incubation with indirubin-3'-oxime prevented the release of calcium by MPT induction (Fig. 6).

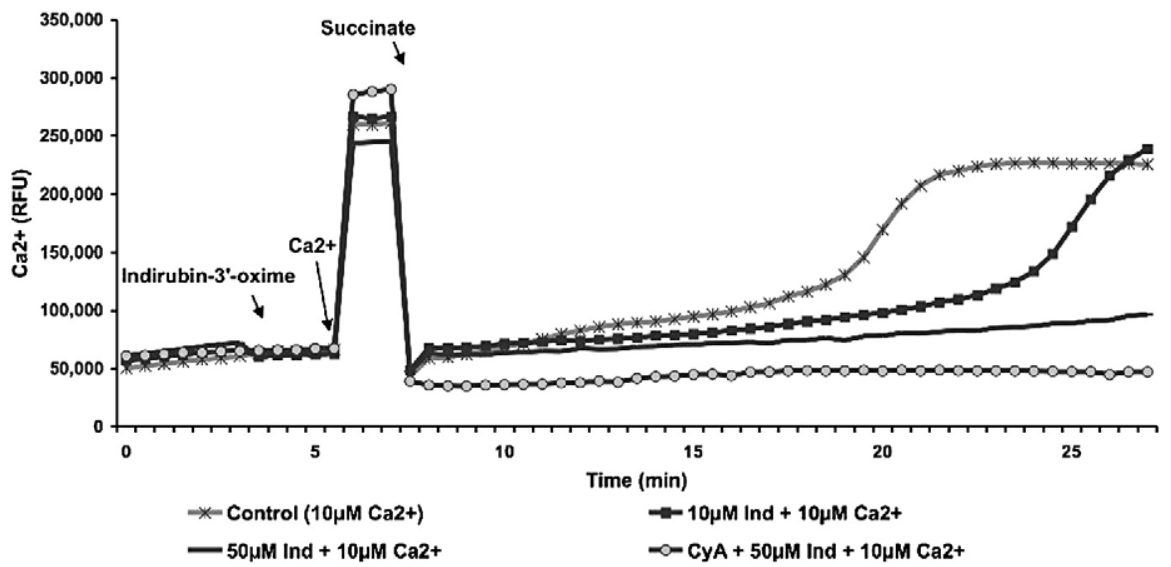

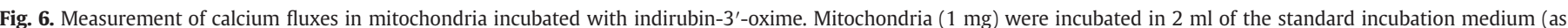

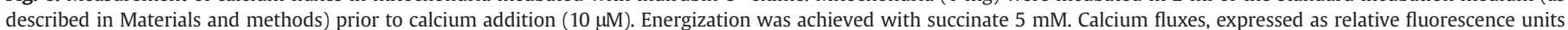

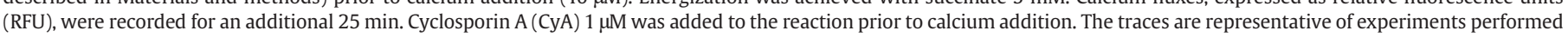
with mitochondria pre-incubated with different indirubin-3'-oxime concentrations. 


\section{Discussion}

The study of drug-induced mitochondrial toxicity may elucidate distinct mechanisms by which drugs interfere with energy production by the cell. The indirubin family of compounds has been associated with putative therapeutic potential as an anti-cancer agent and in the development of therapies for diabetes and Alzheimer's disease (Hoessel et al., 1999; Leclerc et al., 2001; Shi and Shen, 2008). Mitochondria play a key role in energy production, calcium homeostasis, generation of free radicals and apoptosis (Bernardi and Rasola, 2007; Orrenius, 2007). ATP synthesis by the mitochondrial oxidative phosphorylation system is required to maintain cell viability since almost all intracellular ATP is generated in the mitochondria and about one-third of the cellular adenine nucleotides are located in these organelles. Thus, disturbance of mitochondrial function has a variety of functional consequences and is found in most human diseases, including neurodegenerative diseases, stroke, cardiovascular disorders, ischemia/reperfusion, and cancer (Wang et al., 2005; Hunt et al., 2006; Halestrap et al., 2007; Kim et al., 2008). In this context, damage to mitochondria can be a limiting factor in clinical settings, disabling a potential therapeutic action of promising drugs. The aim of this study was to evaluate if indirubin-3'-oxime is a mitochondrial toxicant by interfering with the bioenergetic features of the mitochondria.

Our results demonstrate that, in isolated rat liver mitochondria, indirubin-3'-oxime impairs the oxidative phosphorylation capacity as shown by decreased respiratory control ratio and ADP/O. Moreover, indirubin-3'-oxime depresses both mitochondrial $\Delta \Psi$ and state 3 respiration but induces stimulation of state 4 respiration. The observed effects show that concentrations of indirubin-3'-oxime higher than $10 \mu \mathrm{M}$ affect mitochondrial function in a similar way. The decrease in both state 3 respiratory rates and FCCP-induced oxygen consumption indicated a loss of oxidative capacity in mitochondria incubated with indirubin-3'-oxime. The decrease in oxygen consumption reflects an inhibition of the maximal velocity of electron flow from reduced substrates to oxygen in mitochondria incubated with indirubin-3'-oxime, indicating a behavior of classical inhibitors of the electron transport chain. As shown by the decrease in the activities of the complexes of the electron transport chain, succinate dehydrogenase and cytochrome $c$ oxidase, indirubin-3'-oxime inhibits both substrate oxidation and oxygen consumption, directly affecting the activity of individual complexes of the electron transport chain, thereby decreasing the electrical potential across the inner mitochondrial membrane. Additionally, indirubin-3'oxime-induced increase in state 4 respiration, which paralleled the partial collapse of the $\Delta \Psi$, probably reflects an uncoupling effect of this drug on the oxidative phosphorylation system, resulting in an increase in the permeability of the mitochondrial inner membrane to protons (proton leak).

The increase in the lag phase and the decrease in the ADP/O ratio in mitochondria incubated with indirubin-3'-oxime indicated an uncoupling between the oxidation of reduced substrates by the electron transport chain and the phosphorylation of ADP to ATP by the ATPsynthase. This decrease in the phosphorylative efficiency was associated with an inhibitory action of indirubin directly on the ATPsynthase. One interesting finding regarding the effects of indirubin-3'-oxime on isolated mitochondria was that although the impairment of oxidative phosphorylation, this compound had a partial preventive role on calcium-induced MPT. MPT induction disrupts the permeability barrier of the inner membrane, thus dissipating the membrane potential and $\mathrm{pH}$ gradient that together drive ATP synthesis through oxidative phosphorylation (Lemasters, 2007). The MPT is induced under conditions of high matrix calcium, especially when this is accompanied by oxidative stress, high phosphate and low adenine nucleotide concentrations (Lemasters, 2007). Since indirubin- $3^{\prime}$-oxime decreases the $\Delta \Psi$ and the mitochon- drial uptake of calcium is dependent on $\Delta \Psi$, indirubin-3'-oxime partial protective action on calcium-induced MPT could be the result of the prevention of excessive mitochondrial calcium accumulation. However, mitochondrial calcium uptake was not affected by incubation of mitochondria with indirubin-3'-oxime. The observation that the combination ADP plus indirubin-3'-oxime have a stronger protective effect on calcium-induced MPT, may suggest that indirubin-3'-oxime also interacts with components of the MPT thereby affecting its induction.

In conclusion, our results demonstrate that indirubin-3'-oxime has direct effects on mitochondrial function by disturbing the oxidative phosphorylative efficiency that may lead to mitochondrial failure in exposed cells. Work conducted by MacDonald and coworkers has shown that in cells in culture exposed to indirubin for $1 \mathrm{~h}$, there is a $90 \%$ decrease in $\Delta \Psi$, as well as inhibition of cell proliferation (MacDonald et al., 2006). While our manuscript was in preparation, Shi and Shen have shown that indirubin-3'-monoxime (in the range of concentrations that affect mitochondrial bioenergetics) induces type II cell apoptosis in HeLa cells with the critical involvement of the proapoptotic proteins such as Bid and Bax at the level of mitochondria (Shi and Shen, 2008). This work has drawn attention to an unrecognized action of indirubin-3'-oxime on mitochondria, suggesting a direct effect of the compound on mitochondrial function.

\section{Acknowledgments}

A. P. Rolo, J. P. Teodoro and F. V. Duarte are recipients of a fellowship from Science and Technology Foundation (SFRH/BPD/26514/2006, SFRH/BD/38467/2007 and SFRH/BD/38372/2007, respectively). This work was supported by Science and Technology Foundation grant PTDC/SAU-OSM/72443/2006.

\section{References}

Barillas, R., Friehs, I., Cao-Danh, H., Martinez, J.F., del Nido, P., 2007. Glycogen synthase kinase-3 $\beta$ improves tolerance to ischemia in hypertrophied hearts. Ann. Thorac Surg. 84, 126-133.

Bernardi, P., Rasola, A., 2007. Calcium and cell death: the mitochondrial connection. Subcell. Biochem. 45, 481-506.

Brautigan, D.L., Ferguson-Miller, S., Margoliash, E., 1978. Mitochondrial cytochrome $c$ : preparation and activity of native and chemically modified cytochrome $c$. Methods Enzymol. 53, 128-133.

Eisenbrand, G., Hippe, F., Jakobs, S., Muehlbeyer, S., 2004. Molecular mechanisms of indirubin and its derivates: novel anticancer molecules with their origin in traditional Chinese phytomedicine. J. Cancer Res. Clin. Oncol. 130, 627-635.

Estabrook, R.W., 1967. Mitochondrial respiratory control and the polarographic measurements of ADP/O ratios. Methods Enzymol. 10, 41-47.

Gazotti, P., Malmstrom, K., Brdiczka, D., 1979. In: Carafoli, E., Semenza, G. (Eds.), Membrane Biochemistry: A Laboratory Manual on Transport And Bioenergetics. Springer-Verlag, New York, pp. 6-69.

Gornall, A.G., Bardawill, C.J., David, M.M., 1949. Determination of serum proteins by means of the biuret reaction. J. Biol. Chem. 177, 751-766.

Halestrap, A.P., Clarke, S.J., Khaliulin, I., 2007. The role of mitochondria in protection of the heart by preconditioning. Biochim. Biophys. Acta 1767, 1007-1031.

Hoessel, R., Leclerc, S., Endicott, J.A., Nobel, M.E.M., Lawrie, A., Tunnah, P., Leost, M. Damies, E., Marie, D., Marko, D., Niederberger, E., Tang, W., Eisenrand, G., Meijer, L. 1999. Indirubin, the active constituent of a Chinese antileukaemia medicine, inhibits cyclin-dependent kinases. Nat. Cell Biol. 1, 60-67.

Hunt, N.D., Hyun, D.H., Allard, J.S., Minor, R.K., Mattson, M.P., Ingram, D.K., de Cabo, R., 2006. Bioenergetics of aging and calorie restriction. Ageing Res. Rev. 5, 125-143.

Jope, R.S., Yuskaitis, C.J., Beurel, E., 2007. Glycogen synthase kinase-3 (GSK3): inflammation. Diseases and Therapeutics Neurochem. Res. 32, 577-595.

Kamo, N., Muratsugu, M., Hongoh, R., Kobatake, V., 1979. Membrane potential of mitochondria measured with an electrode sensitive to tetraphenylphosphonium and relationship between proton electrochemical potential and phosphorylation potential in steady state. J. Membr. Biol. 49, 105-121.

Kim, J.A., Wei, Y., Sowers, J.R., 2008. Role of mitochondrial dysfunction in insulin resistance. Circ. Res. 102, 401-414.

Leclerc, S, Garnier, M, Hoessel, R, Marko, D, Bibb, J.A, Snyder, G.L, Greengard, P Biernat, J., Wu, Y.Z., Mandelkowl, E.M., Eisenbrand, G., Meijer, L., 2001. Indirubins inhibit glycogen synthase kinase-3 $\beta$ and CDK5/P25, two protein kinases involved in abnormal Tau phosphoryltion in Alzheimer's disease-a property common to most cyclin-dependent kinase inhibitors? J. Biol. Chem. 276, 251-260.

Lee, J.W., Moon, M.J., Min, H.Y., Chung, H.J., Park, E.J., Park, H.J., Hong, J.Y., Kim, Y.C., Lee, S. K., 2005. Induction of apoptosis by a novel indirubin-5-nitro-3'-monoxime, a CDK inhibitor, in human lung cancer cells. Bioorg. Med. Chem. Lett. 15, 3948-3952. 
Lemasters, J.J., 2007. Modulation of mitochondrial membrane permeability in pathogenesis, autophagy and control of metabolism. J. Gastroenterol. Hepatol. 22 (Suppl 1), 531-537.

MacDonald, M.L., Lamerdin, J., Owens, S., Keon, B.H., Bilter, G.K., Shang, Z., Huang, Z., Yu, H., Dias, J., Minama, T., Michnick, S.W., Westwick, J.K., 2006. Identifying offtarget effects and hidden phenotypes of dugs in human cells. Nat. Chem. Biol. 2, 329-337.

Meijer, L., Skaltsounis, A.L., Magiatis, P., Polychronopoulos, P., Knockaert, M., Leost, M., Ryan, Z.P., Vonica, C.A., Brivanlou, A., Dajani, R., Crovace, C., Tarricone, C., Musacchio A., Roe, S.M., Pearl, L., Greengard, P., 2003. GSK-3-Selective Inhibitors derived from Tyrian purple indirubins. Chem. Biol. 10, 1255-1266.

Orrenius, S., 2007. Reactive oxygen species in mitochondria-mediated cell death. Drug Metab. Rev. 39, 443-455.

Palmeira, C.M., Wallace, K.B., 1997. Benzoquinone inhibits the voltage-dependent induction of the mitochondrial permeability transition caused by redox-cycling naphtoquinones. Toxicol. Appl. Pharmacol. 143, 338-347.

Palmeira, C.M., Moreno, A.J., Madeira, V.M.C., 1994. Interactions of herbicides 2,4-D and dinoseb with liver mitochondrial bioenergetics. Toxicol. Appl. Pharmacol. 127, 50-57.
Rolo, A.P., Oliveira, P.J., Moreno, A.J., Palmeira, C.M., 2000. Bile acids affect liver mitochondrial bioenergetics: possible relevance for cholestasis therapy. Toxicol. Sci. 57, 177-185.

Sato, N., Meijer, L., Skaltsounis, L., Greengard, P., Brivanlou, A.H., 2004. Maintenance of pluripotency in human and mouse embryonic stem cells through activation of Wnt signalling by a pharmacological GSK-3-specific inhibitor. Nat. Med. 10, 55-63.

Shi, J., Shen, H.-M., 2008. Critical role of Bid and Bax in indirubin-3'-monoxime-induced apoptosis in human cancer cells. Biochem. Pharmacol. 75, 1729-1742.

Singer, T.P., 1994. Determination of the activity of succinate, NADH, choline and glycerophosphate dehydrogenases. Biochim. Biophys. Acta. 1363, 100-124.

Rajdev, S., Reynolds, I.J., 1993. Calcium green-5N, a novel fluorescent probe monitoring high intracellular free $\mathrm{Ca}^{2+}$ concentrations associated with glutamate excitotoxicity in cultured rat brain neurons. Neurosc. Lett. 162, 149-152.

Wang, J., Xiong, S., Xie, C., Markesbery, W.R., Lovell, M.A., 2005. Increased oxidative damage in nuclear and mitochondrial DNA in Alzheimer's disease. J. Neurochem. 93, 953-962.

Zhang, N., Jiang, Y., Zou, J., Zhang, B., Jin, H., Wang, Y., Yu, Q., 2006. 3D QSAR for GSK-3ß inhibition by indirubin analogues. Eur. J. Med. Chem. 41, 373-378. 